\title{
Impact of Training and Municipal Support on Primary Health Care-Based Measurement of Alcohol Consumption in Three Latin American Countries: 5-Month Outcome Results of the Quasi-experimental Randomized SCALA Trial
}

\author{
Peter Anderson, MD ${ }^{7,2}$ (1) , Jakob Manthey, MSc ${ }^{3,4}$, Eva Jané Llopis, PhD ${ }^{1,5,6}$, \\ Guillermina Natera Rey, $P h D^{7}$, Ines V. Bustamante, $P h D^{8}$, Marina Piazza, MPH${ }^{8}$, \\ Perla Sonia Medina Aguilar, $P h D^{7}$, Juliana Mejía-Trujillo, MSc?', \\ Augusto Pérez-Gómez, PhD9, Gill Rowlands, MD², Hugo Lopez-Pelayo, PhD 10,11,12, \\ Liesbeth Mercken, $\mathrm{PhD}^{7}$, Dasa Kokole, MSc' , Amy O'Donnell, PhD', \\ Adriana Solovei, $M S c^{7}$, Eileen Kaner, $P h D^{2}$, Bernd Schulte, $P h D^{4}$, Hein de Vries, $P h D^{7}$, \\ Christiane Schmidt, Dip Psych ${ }^{4}$, Antoni Gual, MD 10,11,12, and Jürgen Rehm, PhD 3,6, 13,14,15
}

\begin{abstract}
'Department of Health Promotion, CAPHRI Care and Public Health Research Institute, Maastricht University, Maastricht, The Netherlands; ${ }^{2}$ Population Health Sciences Institute, Newcastle University, Newcastle upon Tyne, UK; ${ }^{3}$ Institute for Clinical Psychology and Psychotherapy, TU Dresden, Dresden, Germany; ${ }^{4}$ Center for Interdisciplinary Addiction Research (ZIS), Department of Psychiatry and Psychotherapy, University Medical Center Hamburg-Eppendorf, Hamburg, Germany; ${ }^{5}$ Univ. Ramon Llull, ESADE, Barcelona, Spain; 6 Institute for Mental Health Policy Research, CAMH, Toronto, ON, Canada; ${ }^{7}$ Instituto Nacional de Psiquiatría Ramón de la Fuente Muñiz, Ciudad de México, CDMX, Mexico; ${ }^{8}$ School of Public Health and Administration, Universidad Peruana Cayetano Heredia, Lima, Peru; ${ }^{9}$ Corporación Nuevos Rumbos, Bogotá, Colombia; ${ }^{10}$ Addictions Unit, Psychiatry Dept., Hospital Clínic, Barcelona, Spain; ${ }^{11}$ Red de Trastornos Adictivos, Instituto Carlos III, Madrid, Spain; ${ }^{12}$ Institut d'Investigacions Biomèdiques August Pi Sunyer (IDIBAPS), Barcelona, Spain; ${ }^{13}$ Dalla Lana School of Public Health, University of Toronto, Toronto, ON, Canada; ${ }^{14}$ Department of Psychiatry, University of Toronto, Toronto, ON, Canada; ${ }^{15}$ Department of International Health Projects, Institute for Leadership and Health Management, I.M. Sechenov First Moscow State Medical University, Moscow, Russian Federation.
\end{abstract}

PURPOSE: We aimed to test the effects of providing municipal support and training to primary health care providers compared to both training alone and to care as usual on the proportion of adult patients having their alcohol consumption measured.

METHODS: We undertook a quasi-experimental study reporting on a 5-month implementation period in 58 primary health care centres from municipal areas within Bogotá (Colombia), Mexico City (Mexico), and Lima (Peru). Within the municipal areas, units were randomized to four arms: (1) care as usual (control); (2) training alone; (3) training and municipal support, designed specifically for the study, using a less intensive clinical and training package; and (4) training and municipal support, designed specifically for the study, using a more intense clinical and training package. The primary outcome was the cumulative proportion of consulting adult patients out of the population registered within the centre whose alcohol consumption was measured (coverage).

Protocol Version: Final version, 25 February 2020. Jané Llopis E, Anderson P, Piazza $M$ et al. Implementing primary health care-based measurement, advice and treatment for heavy drinking and comorbid depression at the municipal level in three Latin American countries: final protocol for a quasi-experimental study (SCALA study). BMJ Open. In Press.

Received July 31, 2020

Accepted December 17, 2020

Published online January 19, 2021
RESULTS: The combination of municipal support and training did not result in higher coverage than training alone (incidence rate ratio $(\mathrm{IRR})=1.0,95 \% \mathrm{CI}=0.6$ to 0.8 ). Training alone resulted in higher coverage than no training (IRR $=9.8,95 \% \mathrm{CI}=4.1$ to 24.7 ). Coverage did not differ by intensity of the clinical and training package (coefficient $=0.8,95 \%$ CI 0.4 to 1.5 ).

CONCLUSIONS: Training of providers is key to increasing coverage of alcohol measurement amongst primary health care patients. Although municipal support provided no added value, it is too early to conclude this finding, since full implementation was shortened due to COVID19 restrictions.

TRIAL REGISTRATION: Clinical Trials.gov ID: NCT03524599; Registered 15 May 2018; https:// clinicaltrials.gov/ct2/show/NCT03524599

KEY WORDS: primary health care; municipal action; heavy drinking; Institute for Health Care Improvement; implementation; measurement of alcohol consumption; AUDIT-C; brief advice; Colombia; Peru; Mexico.

$\mathrm{J}$ Gen Intern Med 36(9):2663-71

DOI: $10.1007 / \mathrm{s} 11606-020-06503-9$

(C) The Author(s) 2021

\section{INTRODUCTION}

Alcohol use is a leading risk factor for ill-health and premature death, increasing a wide range of cancers, and cardiovascular and gastrointestinal diseases ${ }^{1-4}$. Within the World Health Organization's (WHO) SAFER initiative, facilitating population- 
level health service access to measurement of alcohol consumption, and delivering brief advice and treatment as required, is one of five high-impact strategies to reduce the harm done by alcohol ${ }^{5}$.

A number of meta-analyses and systematic reviews have demonstrated the impact ${ }^{6-8}$ and cost-effectiveness ${ }^{9,10}$ of primary health care (PHC)-based measurement and brief advice programmes in reducing heavy drinking.

Despite the evidence of impact, few governments seeming willing to undertake the necessary investments to ensure countrywide implementation, and the global penetration of such programmes remains generally very low ${ }^{10-16}$. While no published data is available of the extent of PHC-based measurement and brief advice activity in reducing heavy drinking in the three study countries of the SCALA trial (Colombia, Mexico, and Peru), the country investigators report that such work is not normally undertaken.

Lack of motivation, being too busy, and lack of adequate training and support materials are expressed as important barriers to the delivery of measurement and brief advice programmes ${ }^{17}, 18$. Conversely, having more time, having less intense programmes to deliver, and having more active patients asking for alcohol advice have been expressed as facilitators for implementing measurement and brief advice ${ }^{19,20}$.

Within the field of implementation science ${ }^{21}$, systematic reviews $^{22,23}$ and multi-country studies ${ }^{24-27}$ have demonstrated the importance of providing training to PHC providers in increasing their activity in measuring alcohol consumption and giving brief advice to identified heavy drinkers to help reduce their alcohol consumption. It has been widely proposed that PHC-based activities within complex health systems could be improved by addressing underlying structural and support factors ${ }^{28}$, embedding PHC-based measurement and brief advice programmes within the frame of supportive community and municipal environments ${ }^{29-33}$.

The international SCALA project (Scale-up of Prevention and Management of Alcohol Use Disorders in Latin America, www.scalaproject.eu) aims to evaluate the impact of multilevel interventions on rates of health care-based measurement, advice and treatment for heavy drinking of alcohol and comorbid depression ${ }^{34,35}$ (although, in this paper, we only report results for alcohol).

Latin American countries are chosen for three reasons: the regional importance of alcohol as a risk factor for morbidity and premature death ${ }^{36}$; health system reforms that emphasize primary health care as a vehicle to achieve universal health coverage and prevention ${ }^{37-39}$; and fast track research from high-income countries to Latin American middle-income countries $^{40-46}$.

We test the following three pre-published hypotheses ${ }^{35}$ :

Hypothesis 1: For PHC centres whose providers have received training, the presence of municipal support, designed and implemented for the purpose of the SCALA study, leads to more sustainable coverage of alcohol measurements than the absence of municipal support;

Hypothesis 2: In the absence of municipal support, PHC centres whose providers receive training obtain higher coverage than PHC centres whose providers have not received training; and

Hypotheses 3: In the presence of municipal support, use of a less intense version of a delivered clinical package and training does not lead to less coverage of alcohol measurements than delivery of a standard more intense clinical package and training.

There are deviations to the protocol ${ }^{35}$, due to COVID-19 illness. Our original plan was for an 18-month implementation period with formal evaluation points at 6,12 , and 18 months. According to the WHO COVID-19 dashboard ${ }^{47}$, Colombia, Mexico, and Peru have been amongst some of the worst affected countries in the world for COVID-19. Due to the social distancing and 'lock-down' measures implemented in the countries as a mitigation response to COVID-19 illness, much routine preventive work was halted and many providers were confined to home. This compromised continued measurement and advice activities as part of SCALA, and an inability for the local researchers to visit the PHC centres. Thus, we had to pause the work and we chose to bring forward the first 6-month evaluation time point to 5 months, given restrictions on preventive work beyond COVID-19 and on the uncertainty as to when and how full implementation might restart.

\section{METHODS}

The study is a quasi-experimental design, ${ }^{48}$ comparing changes in measurement of alcohol consumption between PHC centres in intervention municipal areas and PHC centres in similar control municipal areas (Fig. 1).

One intervention municipal area was investigator-selected from each of Bogotá (Colombia), Mexico City (Mexico), and Callao-Lima (Peru). One control municipal area was investigator-selected from each of the same cities, on the basis of comparability in terms of socio-economic characteristics, and with sufficient geographical separation to minimize spillover effects from the intervention municipal area. Randomized selection of the municipal areas was not feasible due to organizational limitations, and the need to obtain approval from municipal authorities.

Within the municipal areas, the units of allocation and analysis, i.e., study participants, are PHC centres and the providers working in them. Centres were invited to join the study until a minimum of 27 were achieved within each of the two municipal areas (intervention and control) across the three countries (nine per municipal area within each of the three countries). Within each centre, eligible providers include any fully trained health care provider, with written informed consent for participation. In the end, 58 centres were recruited, 29 


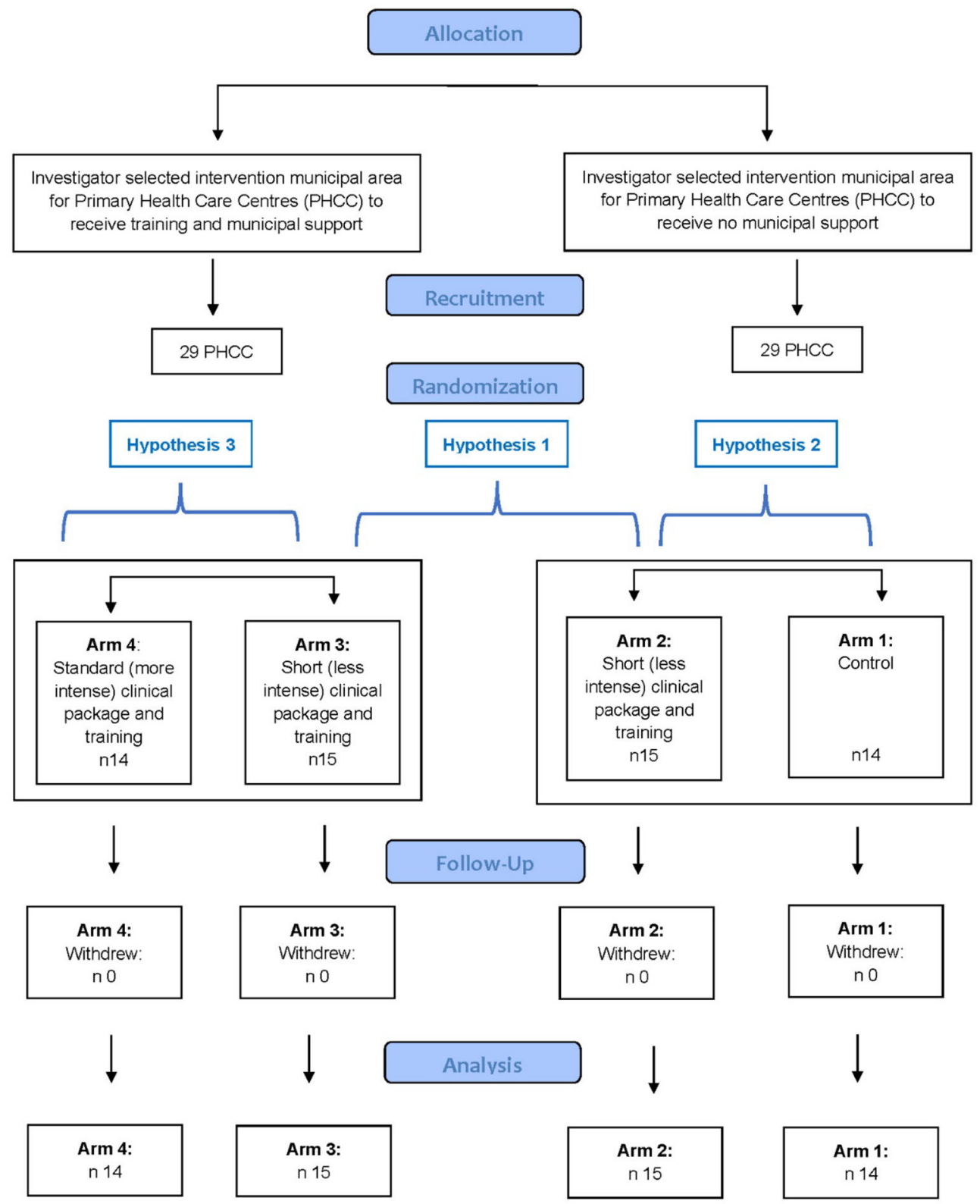

Figure 1 Study flow chart.

in the intervention municipal area and 29 in the control municipal area.

Within the control municipal area, 14 centres were randomly allocated to control (arm 1), and 15 to receive less intense training to implement a less intense clinical package (arm 2). Within the intervention municipal area, in which municipal support was provided, 15 centres were randomly allocated to receive less intense training to implement a less intense clinical package (arm 3), and 14 were allocated to receive standard (more intense) training to implement a standard (more intense) clinical package (arm 4). Random allocation was stratified by country and undertaken using Excel random number generator.

The clinical package, tailored for local use, comprised measurement instruments, patient information and advice material, and provider guidance material, with the differences between the standard (more intense) and less intense clinical materials described in the published protocol ${ }^{35}$. The less intense version was a simplified version of standard UK-based materials ${ }^{49}$, deliverable in a reduced period of time, noting evidence that the duration of brief advice has little impact on outcome $^{7,50,51}$.

All providers in arms 2-4 were given one and a half to $2 \mathrm{~h}$ tailored training in arms 2 and 3, and three and a half to $4 \mathrm{~h}$ tailored training in arm 4 , after the baseline measurement period and before the implementation period. Training focussed on practical skills in undertaking alcohol measurement and in delivering brief advice, and on using the measurement instruments. The difference between arm 4 and arms 2 and 3 was to test hypothesis 3 (less intense clinical intervention and training does not lead to less coverage of alcohol measurements than the use of standard more intense clinical 
intervention and training). The training methods were the same across all three arms, using a modelling strategy based on videos, and tailored to the PHC settings and systems in the three Latin American countries.

In all arms, PHC providers were asked to measure the alcohol consumption of all adult patients who consulted for any reason using AUDIT- $\mathrm{C}^{52}$, with a stem question to exclude patients who had already completed the AUDIT-C during the study period. The three AUDIT-C questions were included in a paper tally sheet completed by the provider, in which the provider documented the outcome of the consultation (advice given, patient referred etc.).

The municipal support inputs to arms 3 and 4, based on the Institute for Healthcare Improvement's frame for going to scale $^{28}$, were designed, tailored, and implemented for the purposes of the SCALA study. They included five common components across the three countries ${ }^{35}$ :

i. Creation of local stakeholder groups to advise on tailoring, support implementation, and review drivers of successful action;

ii. Appointment of local project champions to advocate for successful implementation;

iii. Implementation of five evidence-based adoption mechanisms;

iv. Implementation of five evidence-based support systems; and

v. Implementation of community-based communication campaigns.

\section{Data collection}

Data were collected between April 2019 and April 2020. Before the start of baseline data collection, we obtained characteristics of the participating PHC centres, including the number of providers working in the centre by profession and the number of adults registered with the centre (Appendix Table 1). During the course of the study, providers completed the number of adult consultations on a monthly basis. During the baseline and the five implementation months, providers returned completed tally sheets, enabling a calculation of the numbers of adult patients whose alcohol consumption was measured.

\section{Definition of variables for analyses}

All analyses were conducted at the PHC centre level. The primary outcome was the coverage of alcohol measurement, defined as the cumulative proportion of the adult (aged 18+ years) population registered with the PHC centre that has their alcohol consumption measured. The denominator definitions of registered population by country are summarized in Appendix Table 1 . The nominator of the primary outcome was calculated by summing up the number of completed measurement instruments (AUDIT-C) per PHC Centre and month, before computing a cumulative variable of alcohol measurements across all months per PHC centre and month. The nominator measures the number of individual patients whose alcohol consumption was measured, rather than patient encounters. The final primary outcome variable is expressed as the cumulative number of alcohol measurements per 1000 registered patients. Two adjustment variables were considered: (1) dummy-coded country variables, to account for differences between countries; and (2) baseline coverage, to account for variations in measurement activity before training and municipal support were implemented. All descriptive and inferential analyses of the primary outcome were weighted for PHC Centre size, i.e., for the number of adults registered with the Centre. The weight variable was normalized by dividing the Centre size by the mean Centre size across all Centres.

\section{Power calculations}

As detailed in the protocol ${ }^{35}$, amongst centres that did not receive municipal support, the planned study was powered to detect a doubling of cumulative coverage after 12 months from $3.25 \%$ of the registered adult population in control centres to $7.5 \%$ in centres whose providers had received training ( $82 \%$ power at a significance level of $5 \%$ ). In the presence of training, the study was powered to detect a further doubling of cumulative coverage after 12 months from $7.5 \%$ in centres that did not receive municipal support to $15 \%$ in centres that did receive municipal support $(96.5 \%$ power at a significance level of $5 \%)^{53}$.

\section{Statistical analyses}

The distribution of the primary outcome is best described as negative binomial, Appendix Figure 1. For testing the three hypotheses, the primary outcome was analysed cross-sectionally using cumulative data at month 5, with negative binomial regressions comparing the dependent variable by exposure variable, while accounting for country differences and baseline measurement activity. Interaction effects of country and exposure were not significant, Appendix Table 2.

We report exponentiated coefficients of negative binomial regression analyses (with 95\% confidence intervals), such coefficients being incidence rate ratios (IRR). The IRR is the ratio of the outcome (cumulative coverage) for the exposure happening (e.g., training) to the exposure not happening (e.g., no training).

All Peruvian PHC Centres had their fifth month of data collection completed by early February 2020. In Colombia and Mexico, several Centres would have completed their fifth month of data collection only post data closure. For these Centres, the last reported cumulative coverage rate was carried forward, assuming no further measurements, with the estimated impact of missing data being of negligible extent (Appendix Table 3).

Analyses were run with $\mathrm{R}$ version 3.6.1. ${ }^{54}$ 


\section{RESULTS}

The municipal areas were similar in basic demographic variables (Appendix Table 4). At baseline, 58 PHC Centres (Colombia: 20; Mexico: 18; Peru: 20) participated in the study. In total, 622 providers (Colombia: 128; Mexico: 256; Peru: 238) consented to participate; 524 were recruited at baseline and 98 joined the study throughout the implementation period (Table 1).

No Centres dropped out during the study. The median number of registered patients per Centre at baseline was $9048($ mean $=15,006, \mathrm{SD}=23,555)$, with the largest Centre having more than five times more patients registered relative to the second largest $(177,953$ vs 31,501$)$. After removing this outlier from this comparison, the registered patient population size did not differ by arm (ANOVA: $\mathrm{F}=0.528, p=0.655$ ). Across the observed study period, the median number of participating providers per Centre was 7 (mean $=8.7, \mathrm{SD}=$ 6.4), not differing by arm (ANOVA: $\mathrm{F}=2.69, p=0.056$ ), and the median proportion of all providers within a Centre that participated in the study was $35.7 \%$ (mean $=40.4 \%$, SD $=$ $28.3 \%$ ), not differing by arm (ANOVA treating the participation rate as continuous, normally distributed variable: $\mathrm{F}=$ $0.829, p=0.484$ ). The median number of monthly consultations per Centre (averaged over participating providers) was 132 (mean $=191.0, \mathrm{SD}=131.8$ ), not differing by arm (ANOVA: $\mathrm{F}=1.523, p=0.219$ ). The characteristics of the providers by study arm are summarized in Appendix Table 5 .

\section{Training coverage}

The proportion of providers across arms attending at least one training session was $72.3 \%$, being higher in arms 2 and 4 (74.1\% and $76.9 \%$ respectively) than in arm $3(66.3 \%)$ (Appendix Table 5). Any heterogeneity across countries is accounted for by including country as dummy variable in the model.

Table 1 Sample Characteristics

\begin{tabular}{|c|c|c|c|c|}
\hline & $\begin{array}{l}\text { Arm 1 } 1(n \\
=14)\end{array}$ & $\begin{array}{l}\operatorname{Arm} 2(n \\
=15)\end{array}$ & $\begin{array}{l}\operatorname{Arm} 3(n \\
=15)\end{array}$ & $\begin{array}{l}\operatorname{Arm~4(n} \\
=14)\end{array}$ \\
\hline $\begin{array}{l}\text { Total number of } \\
\text { participating } \\
\text { providers }^{\mathrm{a}}\end{array}$ & 126 & 206 & 173 & 144 \\
\hline $\begin{array}{l}\text { Mean number of } \\
\text { providers per } \\
\text { Centre }^{\mathrm{a}}\left(\mathrm{SD}^{\mathrm{c}}\right)\end{array}$ & $7.5(7.4)$ & $10.3(6.3)$ & $8.5(5.9)$ & $8.2(5.7)$ \\
\hline $\begin{array}{l}\text { Mean number of } \\
\text { registered } \\
\text { patients per } \\
\text { Centre }\left(\mathrm{SD}^{\mathrm{c}}\right)\end{array}$ & $\begin{array}{l}23,967.3 \\
(44,346.9)\end{array}$ & $\begin{array}{l}11,164.0 \\
(9936.4)\end{array}$ & $\begin{array}{l}11,616.7 \\
(7674.5)\end{array}$ & $\begin{array}{l}14,020.4 \\
(7961.5)\end{array}$ \\
\hline $\begin{array}{l}\text { Mean number of } \\
\text { consultations } \\
\text { per provider per } \\
\text { month (SD) }\end{array}$ & $\begin{array}{l}181.6 \\
(126.7)\end{array}$ & $\begin{array}{l}179.9 \\
(132.8)\end{array}$ & $\begin{array}{l}203.8 \\
(134.7)\end{array}$ & $\begin{array}{l}198.5 \\
(150.4)\end{array}$ \\
\hline
\end{tabular}

${ }^{a}$ Summed/averaged across baseline and 5-month implementation periods

${ }^{b}$ Some providers in Colombia worked in more than one Centrelarm; thus, the total across all arms is greater than the total number of providers participating in this study

${ }^{c}$ Standard deviation

\section{Municipal action}

In each country, two or three community advisory board meetings of local stakeholders took place and one or two project champions provided ongoing implementation support (Appendix Table 6). Five adoption mechanisms and five support systems were wholly or partly implemented. A communication campaign was initiated in Mexico and Peru during month 4 of implementation and planned for month 6 in Colombia. Due to restrictions of COVID-19, the communication campaign was partially paused or delayed in all three intervention communities. Any heterogeneity across countries is accounted for by including country as dummy variable in the model.

\section{Coverage of alcohol measurements at baseline and month 5 across all arms}

Upon completion of the 1-month baseline assessment, 2.1 per 1000 registered patients had their alcohol consumption measured (SD: 5.4). This baseline level is probably a consequence of all providers being asked to measure the alcohol consumption of all consulting adult patients, rather than an indicator of routine activity, which, prior to SCALA, was considered nonexistent by the country investigators. The cumulative number rose to 11.7 (SD: 22.7) per 1000 after completing the 5-month implementation period.

\section{Evaluation of overall effects}

The distribution of the cumulative coverage at month 5 by hypothesis is summarized in Figure 2 and described for each hypothesis. In Table 2, results of regression analyses testing the three hypotheses are presented. In this table, the coefficient of the exposure variable indicates the incidence rate ratios in measurement rates between the arms as postulated in the respective hypotheses. For example, an incident rate ratio of 9.8 in hypothesis 2 implies that, keeping the covariates constant, PHC centres in arm 2 (providers assigned to be trained), had, on average, a 9.8 times higher alcohol measurement coverage rate than PHC Centres in arm 1 (providers not assigned to be trained). The results are presented for each hypothesis below.

\section{Hypothesis 1: Increased Coverage Through Municipal Support (Arm 3 vs Arm 2). Hypothesis 1 is comparing centres between two investigator-assigned municipal areas. Although the raw cumulative coverage at month 5 was higher in Centres receiving both municipal support and training (mean: 23.9 per 1000, SD: 40.1) relative to Centres receiving training only (mean: 14.1 per 1000, SD: 16.5) (Fig. 2), the difference was not signif- icant (coefficient, incident rate ratio $(\mathrm{IRR})=1.0,95 \% \mathrm{CI}=0.6$ to 1.8 , Table 2). Thus, hypothesis 1 is not confirmed.}

Hypothesis 2: Increased Coverage Through Training (Arm 2 vs Arm 1). Hypothesis 2 is comparing centres randomly allocated to separate arms. The raw cumulative coverage at 


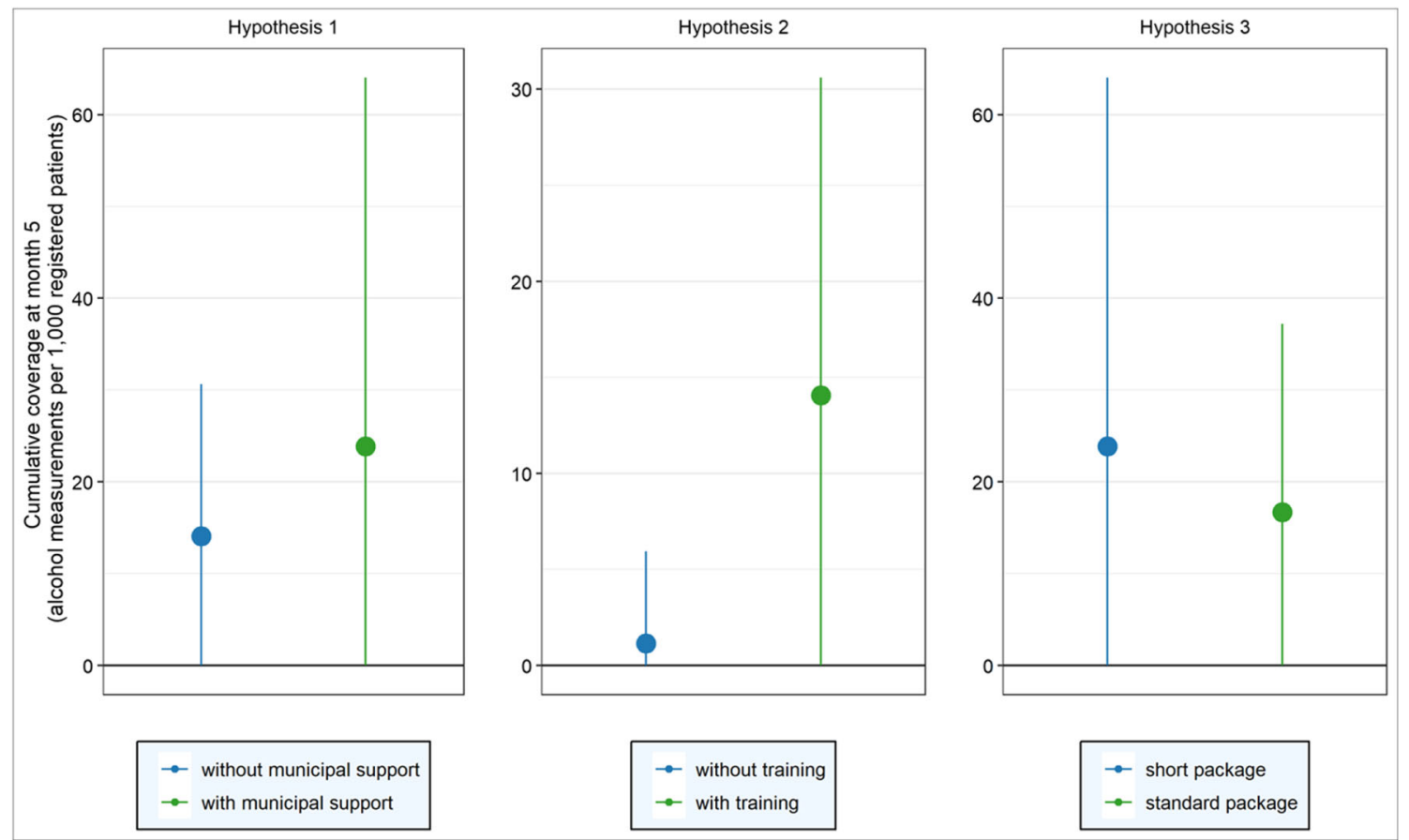

Figure 2 Average cumulative coverage at month 5 for each exposure group, as defined by each hypothesis (points indicate mean and vertical lines indicate mean \pm one standard deviation).

month 5 was higher in PHCUs receiving training (mean: 14.1 per 1000, SD: 16.5) relative to PHCUs not receiving training (1.1 per 1000, SD: 4.8) (Fig. 2), and this difference was significant (coefficient, $\mathrm{IRR}=9.8,95 \% \mathrm{CI}=4.1$ to 24.7 ) (Table 2), that is a 9.8-fold increase in measurement coverage amongst Centres receiving training. Thus, hypothesis 2 is confirmed.

\section{Hypothesis 3: No Increased Coverage Through Longer}

Package (Arm 4 vs Arm 3). Hypothesis 3 is comparing centres randomly allocated to separate arms. Although the raw cumulative coverage at month 5 was higher in Centres working with the less intense package (mean: 23.9 per 1000; SD: 16.7) relative to Centres working with the standard more intense package (16.7 per 1000; SD: 20.5) (Fig. 2), this difference was not significant (coefficient, IRR $=0.8,95 \% \mathrm{CI}=$ 0.4 to 1.5 ) (Table 2). Thus, hypothesis 3 is confirmed.

\section{DISCUSSION}

The SCALA project was set up in middle-income countries to test the following: does training of providers, in the absence of municipal support, lead to a higher cumulative proportion of patients having their alcohol consumption measured (coverage), and, in the presence of training, does the extra provision of municipal-based support lead to improved coverage. We chose adult patient 'coverage' as our outcome measure, since this is similar to the blood pressure model, in which one is interested in the proportion of patients who have had their blood pressure measured ${ }^{55,56}$. Measurement coverage is based on an assumption that advice to cut down is routinely given patients with a high measured alcohol consumption, which, in the ODHIN study, was just under $80 \%{ }^{26}$.

As with the two previous international studies ${ }^{24-27}$, we demonstrated a clear impact of training (hypothesis 2, tested between centres randomly allocated to two separate arms). In the absence of municipal support, centres whose providers received training measured the alcohol consumption of a nearly ten times higher proportion of registered patients (coverage) than centres whose providers had not received

Table 2 Results of Regression Analyses for Evaluating Hypotheses 1-3

\begin{tabular}{llll}
\hline \hline & Hypothesis & Hypothesis & Hypothesis \\
& $\mathbf{1}$ & $\mathbf{2}$ & $\mathbf{3}$ \\
\hline Exposure $^{\mathrm{a}}$ & 1.0 & $9.8^{* *}$ & 0.8 \\
& $(0.6,1.8)$ & $(4.1,24.7)$ & $(0.4,1.5)$ \\
Country (base: Colombia) & $0.3^{* *}$ & 0.4 & \\
Mexico & $(0.1,0.6)$ & $(0.05,2.0)$ & 0.7 \\
& $0.2^{* *}$ & $0.2^{*}$ & $(0.3,1.7)$ \\
Peru & $(0.1,0.5)$ & $(0.04,0.8)$ & $0.3^{* *}$ \\
& $1.05^{*}$ & 1.08 & $1.07 * .7)$ \\
Baseline coverage & $(1.0,1.1)$ & $(0.96,1.4)$ & $(1.0,1.2)$ \\
rate & $42.6^{* *}$ & 3.7 & $23.032^{* *}$ \\
Intercept & $(17.5,123.8)$ & $(1.0,21.0)$ & $(9.2,62.6)$ \\
& 30 & 29 & 29 \\
Observations & -80.8 & -70.5 & -90.1 \\
Log Likelihood & $2.6^{* *}(0.87)$ & $0.9 *(0.43)$ & $1.8^{* *}(0.57)$ \\
Theta & 171.5 & 151.0 & 190.2 \\
Akaike Inf. Crit. & &
\end{tabular}

Presented are exponentiated coefficients of negative binomial regression analyses, which should be interpreted as incidence rate ratios. Numbers in brackets denote $95 \%$ confidence intervals

${ }^{a}$ Exposure variable defined by hypothesis: H1: without (base) vs with municipal support; H2: without (base) vs with training; H3: short (base) vs standard package

$* p<0.1 ; * * p<0.05 ; * * * p<0.01$ 
training, although, as with the previous studies ${ }^{24-27}$, coverage remained small (14/1000 registered population).

Based on the conclusions of the WHO Phase IV study $^{29}$, we had anticipated that municipal support would lead to higher coverage (hypothesis 1, tested between centres between from each of two investigator-assigned municipal areas), but we have not been able to demonstrate this. Municipal support is an action over time that might lead to cumulative effects over time. Furthermore, not all the municipal support interventions have been implemented as planned, in particular the community-based communication campaigns, due to lockdown restrictions following COVID19. For these reasons, we think it premature to conclude that municipal support does not lead to higher coverage.

SCALA is an implementation action study utilizing an evidence-based clinical measurement and advice package. We considered it important to test whether or not coverage was dependent on the clinical package used (standard or less intense). As non-superiority is given (hypothesis 3, tested between centres randomly allocated to two separate arms), a shorter less intense package can be implemented as the norm.

\section{Strengths and Weaknesses}

Our study has a number of strengths. SCALA is the first multicountry primary health care--based study on alcohol that we know of being implemented in middle-income countries. We use coverage of an adult practice population as the primary outcome measure $^{57}$. We based our implementation model on that of the Institute for Health Care Improvement going to scale $^{28}$, and we tailored our materials based on the tailoring for chronic diseases initiative ${ }^{58}$. We have built in mechanisms for replicability and options for exploitation for scale up ${ }^{35}$.

Our study, though, has a number of weaknesses. For one of our hypothesis, the impact of municipal support, we were not able to allocate randomly the two municipal areas. Nevertheless, the two areas were similar in core socio-demographic variables, and the lack of randomization of the areas is unlikely to be the cause of the rejection of the hypothesis. Social distancing measures and lock-down to mitigate the spread of COVID-19 illness meant that we have not been able to complete the full implementation of municipal support. On the other hand, for two of our hypotheses (2 and 3), both of which were confirmed, we were able to allocate randomly the centres between the arms.

While we cannot preclude the possibility of substantially different results based on 6 months of data, testing the hypotheses at an earlier point can be considered more conservative as effects would be expected to increase over time.

We have a number of plans to reset and restart the implementation once lock-downs and social distancing measures are relaxed. We will review and refocus the elements of municipal support, telescoping a sustained and intense action within a short period of time within a second 6-month implementation phase. We will continue to test our original first two hypotheses, but adding additional hypotheses to study the impact of COVID-19 mitigation measures, by comparing results between the first phase (reported here) and the second phase to be implemented. We are also developing telemedicine approaches ${ }^{59}$ for delivering measurement and brief advice for heavy drinking, which we will separately evaluate.

\section{Implications}

There are a number of practice and policy implications. We propose the use of coverage as the main outcome measure for similar implementation studies. Tailoring indicated the importance of keeping clinical and training packages as short and as simple as possible to enable widespread deployment. To increase coverage of alcohol measurement (and subsequent advice to identified heavy drinkers), health care providers require tailored skills-based training. This can be of relative short duration ( $2 \mathrm{~h}$ ). Although we did not find added value of municipal support, we consider it premature to dismiss the need for municipal support, as disruption due to social distancing measures and lock-downs to mitigate spread of COVID-19 illness has not allowed us to test the full sustainable impact of municipal support as planned.

To deliver widespread implementation beyond the test phase, ministries of health at municipal and country levels are represented in the Community Advisory Boards created in each intervention municipality to ensure sustainability and to facilitate scale-up at municipal and country levels. ${ }^{35}$ SCALA works closely with the Pan American Health Organization (PAHO), with the principal investigator from Mexico being a Collaborating Centre with $\mathrm{PAHO}$, to facilitate scale-up at Latin American levels.

Corresponding Author: Peter Anderson, MD; Population Health Sciences Institute, Newcastle University, Newcastle upon Tyne, UK (e-mail: peter.anderson@maastrichtuniversity.nl).

Supplementary Information The online version contains supplementary material available at https://doi.org/10.1007/s11606-02006503-9.

\begin{abstract}
Authors' Contributions EJL and PA are the international principal investigators. APG and JMT are the principal investigators in Colombia. GNR and APdL are the principal investigators in Mexico. MP and IVB are the principal investigators in Peru. EJL, PA, MP, AO'D, AG, BS, APG, HdV, GNR, DK, IVB, FB, JMT, AS, APdL, EK, SM, JM, LM, HLP, GR, CS, and JR contributed to the Grant Application for this study. AO'D, BS, CS, and EK designed the clinical package. AG, FB, SM, and HL-P designed the training package and trained the trainers at country level. AS and DK provided input to municipal support. APG, JMT, GNR, APdL, MP, and IVB tailored the packages at country level and trained the PHC providers. PA undertook random allocation generation for the PHCU. APG, JMT, GNR, APdL, MP, and IVB implemented the study at country level and collected the data. JM was data manager and undertook the data analyses. PA and JM drafted the first version of the paper, and revised the paper based on author's feedback and comments. EJL, MP, AO'D, AG, BS, APG, HdV, GNR, DK, IVB, FB, JMT, AS, APdL, EK, SM, LM, HLP, GR, CS, and JR commented on drafts of the manuscript and read and approved the final version.
\end{abstract}

Funding The research leading to these results or outcomes has received funding from the European Horizon 2020 Programme for research, technological development and demonstration under Grant 
Agreement no. 778048 - Scale-up of Prevention and Management of Alcohol Use Disorders and Comorbid Depression in Latin America (SCALA). Participant organizations in SCALA can be seen at: www. scalaproject.eu. The views expressed here reflect those of the authors only and the European Union is not liable for any use that may be made of the information contained therein. H.L.-P. received funding from the Spanish Ministry of Economy and Competitiveness, Instituto de Salud Carlos III through a "Juan Rodés" contract (JR/OOO25, to Dr. López-Pelayo), FEDER. This work is supported by the following institutions: Institut d'Investigacions Biomèdiques August Pi i Sunyer (IDIBAPS), University of Barcelona, Hospital Clinic $i$ Universitari de Barcelona and CERCA Programme / Generalitat de Catalunya. GNR and $A P d L$ received funding from the Fundación Gonzalo Rio Arronte A.C. for the implementation of the project in Mexico. The funders were not involved in the study design. The funders are not be involved in the collection, analysis, interpretation of data, and preparations of any publication.

Data Availability All materials are publicly available on the project website: https://www.scalaproject.eu/. According to the SCALA data management plan, by default, all quantitative datasets generated in the course of the SCALA study will be made openly available through the UK Data Service upon publication of the results (http://www.data-archive.ac.uk/). Prior to publication, all data will be formatted to meet UK Data Service requirements.

\section{Compliance with Ethical Standards:}

Reporting Guidelines: This paper adheres to CONSORT reporting guidelines (http://www.consort-statement.org/).

Ethics Approval and Consent to Participate: The Ethics Committee of the Technical University of Dresden gave final ethical approval for the SCALA project on 12 April 2019, EK90032018. All participating PHC units and participating PHC providers have signed an informed consent form for participation.

Consent for Publication: No individual person's data is published in any form.

Conflict of Interest: The authors declare that they do not have a conflict of interest.

Open Access This article is licensed under a Creative Commons Attribution 4.0 International License, which permits use, sharing, adaptation, distribution and reproduction in any medium or format, as long as you give appropriate credit to the original author(s) and the source, provide a link to the Creative Commons licence, and indicate if changes were made. The images or other third party material in this article are included in the article's Creative Commons licence, unless indicated otherwise in a credit line to the material. If material is not included in the article's Creative Commons licence and your intended use is not permitted by statutory regulation or exceeds the permitted use, you will need to obtain permission directly from the copyright holder. To view a copy of this licence, visit http://creativecommons. org/licenses/by/4.0/.

\section{REFERENCES}

1. Rehm J, Gmel G Sr, Gmel G, et al. The relationship between different dimensions of alcohol use and the burden of disease - an update. Addiction. 2017;112(6):968-1001.

2. GBD 2016 Alcohol Collaborators. Alcohol use and burden for 195 countries and territories, 1990-2016: a systematic analysis for the Global Burden of Disease Study. Lancet. 2018. https://doi.org/10.1016/ S0140-6736(18)31310-2.

3. Wood AM, Kaptoge S, Butterworth AS, Willeit P, et al. Risk thresholds for alcohol consumption: combined analysis of individual-participant data for 599912 current drinkers in 83 prospective studies. Lancet. 2018;391(10129):1513-23.

4. Shield KD, Manthey J, Rylett M, Probst C, Wettlaufer A, Parry CDH, Rehm J. National, regional, and global burdens of disease from 2000 to 2016 attributable to alcohol use: a comparative risk assessment study. Lancet Public Health. 2020;5(1):e51-e61. https://doi.org/10.1016/ S2468-2667(19)30231-2.

5. World Health Organization. SAFER, Alcohol Control Initiative. 2020. https://www.who.int/substance_abuse/safer/en/.

6. O’Donnell A, Anderson P, Newbury-Birch D, Schulte B, Schmidt C, Reimer J, Kaner E. The impact of brief alcohol interventions in primary healthcare: a systematic review of reviews. Alcohol Alcohol. https://doi. org/10.1093/alcalc/agt170.

7. Kaner EF, Beyer FR, Dickinson HO, et al. Effectiveness of brief alcohol interventions in primary care populations. Cochrane Database Syst Rev. 2007;(2):CD004148.

8. Kaner EFS, Beyer FR, Muirhead C, Campbell F, Pienaar ED, Bertholet N, Daeppen JB, Saunders JB, Burnand B. Effectiveness of brief alcohol interventions in primary care populations. Cochrane Database Syst Rev. 2018;(2). Art. No.: CD004148. https://doi.org/10.1002/14651858. CD004148.pub4.

9. Purshouse R, et al. Modelling the cost-effectiveness of alcohol screening and brief interventions in primary care in England. Alcohol Alcohol. 2013;48(2): 180-188.

10. Angus C, Thomas C, Anderson P, Meier PS, Brennan A. Estimating the cost-effectiveness of brief interventions for heavy drinking in PHC across Europe. Eur J Public Health. 2017;27(2):345-51.

11. Colom J, Scafato E, Segura L, et al. Brief interventions implementation on alcohol from the European Health Systems perspective. Front Psychiatry. 2014;5:161.

12. OECD. Tackling Harmful Alcohol Use: Economics and Public Health Policy. OECD Publishing; 2015. http://www.oecd.org/health/tacklingharmful-alcohol-use-9789264181069-en.htm. Accessed 5 Oct 2020

13. Kaner E. Health sector responses. In: Anderson P, Møller L, Galea G, editors. Alcohol in the European Union: Consumption, Harm and Policy Approaches. World Health Organization; 2012. pp. 40-45. Available at: http://www.euro.who.int/__data/assets/pdf_file/0003/160680/ e96457.pdf. Accessed 5 Oct 2020

14. Bendtsen $\mathbf{P}$, Anderson $\mathbf{P}$, Wojnar $\mathbf{M}$, et al. Professional's attitudes do not influence screening and brief interventions rates for hazardous and harmful drinkers: results from ODHIN study. Alcohol Alcohol. 2015;50:430-437.

15. Glass JE, Bohnert KM, Brown RL. Alcohol screening and intervention among United States adults who attend ambulatory healthcare. J Gen Intern Med. 2016;31:739-745.

16. Brown J, West R, Angus C, et al. Comparison of brief interventions in primary care on smoking and excessive alcohol consumption: a population survey in England. Br J Gen Pract. 2016;66:1-9.

17. Wilson GB, Lock CA, Heather $\mathbf{N}$ et al. Intervention against excessive alcohol consumption in primary health care: a survey of GPs' attitudes and practices in England 10 years on. Alcohol Alcohol. 2011;46:570-7.

18. Drummond C,Wolstenholme A, Deluca $\mathbf{P}$, et al. Alcohol interventions and treatment in Europe. In Anderson P, Braddick F, Reynolds J, Gual A (eds). Alcohol Policy in Europe: Evidence from AMPHORA. 2nd edn. The AMPHORA Project. 2013. http://amphoraproject.net/ w2 box/data/e-book/Chapter\%209\%20-\%20AM_E-BOOK_ 2nd\%20edition\%20-\%20July\%202013.pdf. 16 June 2014, date last accessed.

19. McAvoy BR, Donovan RJ, Jalleh G et al. General practitioners, prevention and alcohol-a powerful cocktail? Facilitators and inhibitors of practising preventive medicine in general and early intervention for alcohol in particular: a twelve nation key informant and general practitioner study. Drugs Educ Prev Pol. 2001;8:103-17.

20. Nilsen $\mathbf{P}$, Aalto $\mathbf{M}$, Bendtsen $\mathbf{P}$, Seppä $\mathbf{K}$. Effectiveness of strategies to implement brief alcohol intervention in primary healthcare, Scand J Prim Health Care. 2006;24(1):5-15. https://doi.org/10.1080/02813430500475282.

21. Wensing $\mathbf{M}, \mathbf{G r o l} \mathbf{R}$, Grimshaw J. Improving patient care: the implementation of change in health care, 3. London: Wiley; 2020.

22. Anderson $\mathbf{P}$, Laurant $\mathbf{M}$, Kaner E, Wensing $\mathbf{M}$, Grol R. Engaging general practitioners in the management of hazardous and harmful alcohol consumption: results of a meta-analysis. J Stud Alcohol. 2004;65(2):191-199.

23. Keurhorst $M$, van de Glind I, Bitarello do Amaral-Sabadini M, Anderson P, Kaner E, Newbury-Birch D, Braspenning J, Wensing $\mathbf{M}$, Heinen M, Laurant M. Determinants of successful implementation of screening and brief interventions for hazardous and harmful alcohol 
consumption in primary healthcare. A systematic review and metaregression analysis. Addiction. 2015;110:877-900.

24. Anderson P, Kaner E, Wutzke S, Funk M, Heather N, Wensing M, Grol R, Gual A, Pas L. Attitudes and managing alcohol problems in general practice: an interaction analysis based on findings from a WHO collaborative study. Alcohol Alcohol. 2004;39(4):351-356.

25. Funk M, Wutzke S, Kaner E, et al. A multicountry comparator led trial of strategies to promote dissemination and implementation of brief alcohol intervention in PHC: findings of a World Health Organization collaborative study. J Stud Alcohol. 2005;66(3):379-388. https://doi. org/10.15288/jsa.2005.66.379.

26. Anderson P, Bendsten P, Spak F, Reynolds J, Drummond C, Segura L, Keurhorst MN, Palacio-Vieira J, Wojnar M, Parkinson K, Colom J, Kłoda K, Deluca P, Baena B, Newbury-Birch D, Wallace P, Heinen M, Wolstenholme A, van Steenkiste B, Mierzecki A, Okulicz- Kozaryn K, Ronda G, Kaner E, Laurant M, Coulton S, Gual T. Improving the delivery of brief interventions for heavy drinking in PHC: outcome results of the ODHIN five country cluster randomized factorial trial. Addiction. 2016. https://doi.org/10.1111/add.13476.

27. Anderson $\mathbf{P}$, Gual T, Coulton S, Kaner E, Bendsten $\mathbf{P}$, Kłoda $\mathbf{K}$, Reynolds J, Keurhorst MN, Segura, Wojnar M, Mierzecki A, Deluca P, Newbury-Birch D, Parkinson K, Okulicz- Kozaryn $\mathrm{K}$, Drummond C, Laurant M. Improving the delivery of brief interventions for heavy drinking in PHC: nine month outcomes of the ODHIN five country cluster randomized factorial trial. Ann Fam Med. 2017;15:335-340. https://doi. org/10.1370/afm.2051.

28. Barker PM, Reid A, Schall Mw. A framework for scaling up health interventions: lessons from large-scale improvement initiatives in Africa. Implement Sci. 2016;11(1):12. https://doi.org/10.1186/s13012-0160374-x4731989.

29. Heather N. editor. WHO Collaborative Project on Identification and Management of Alcohol-related Problems in PHC - Report to the World Health Organisation on Phase IV: Development of Country-wide Strategies for Implementing Early Identification and Brief Intervention in PHC Geneva: World Health Organisation, Department of Mental Health and Substance Abuse; 2006. http://www.who.int/substance_abuse/publications/identification_management_alcoholproblems_phaseiv. pdf. Accessed 5 Oct 2020

30. Keurhorst $\mathbf{M}$, Heinen $\mathbf{M}$, Colom $\mathbf{J}$ et al. Strategies in primary healthcare to implement early identification of risky alcohol consumption: why do they work or not? A qualitative evaluation of the ODHIN Study. Keurhorst et al. BMC Fam Pract. 2016;17:70. https://doi.org/10.1186/s12875016-0461-8.

31. Babor T, Del Boca F, Bray JW. Screening, brief intervention and referral to treatment: implications of SAMHSA's SBIRT initiative for substance abuse policy and practice. Addiction. 2017;112(Supple. 2):110-117.

32. Vendetti J, Gmyrek A, Damon D, Singh M, McRee B, Del Boca F. Screening, brief intervention and referral to treatment (SBIRT): implementation barriers, facilitators and model migration. Addiction. 2017;112(Suppl. 2):23-33.

33. Singh M, Gmyrek A, Hernandez A, Damon D, Hayashi S. Sustaining screening, brief intervention and referral to treatment (SBIRT) services in health-care settings. Addiction. 2017;112 (Suppl. 2):92-100.

34. Boden JM, Fergusson DM. Alcohol and depression. Addiction. 2011;106:906-14.

35. Jané-Llopis E, Anderson P, Piazza M, et al. Implementing primary healthcare-based measurement, advice and treatment for heavy drinking and comorbid depression at the municipal level in three Latin American countries: final protocol for a quasi-experimental study (SCALA study). BMJ Open. 2020;10:e038226. https://doi.org/10.1136/bmjopen-2020-038226.

36. GBD 2015 Risk Factors Collaborators. Global, regional and national comparative risk assessment of 79 behavioural, environmental and occupational, and metabolic risks or clusters of risks, 1990-2015: a systematic analysis for the Global Burden of Disease Study 2015. Lancet. 2016;388: 1659-1724

37. Atun R, Odorico Monteiro de Andrade L, Almeifda G, et al. Health system reform and universal health coverage in Latin America. Lancet. 2015;385: 1230-47.

38. Kruk ME, Porignon D, Rockers PC. The contribution of primary care to health and health systems in low- and middle-income countries: a critica review of major primary care initiatives. Soc Sci Med. 2010;70:904-911.

39. Coylear D, Gomez-Dantes O, Knaul F, et al. Overcoming social segregation in health care in Latin America. Lancet. 2015;385: 1248-59.

40. Serrano CC, Martinez RD, Mendoza MPR, Sánchez JC, Villatoro J. La intervención eficaz del médico general en el tratamiento de bebedores cuyo hábito alcohólico representa un riesgo para su salud o ya les ha ocasionado algún daño. (Preliminary results of a prospective double blind clinical trial). Salud Ment. 1992;15(2).

41. Hoffman KA, Beltrán J, Ponce J, Garcia-Fernandez L, Calderón M, Muench J, et al. Barreras para implementar el despistaje, intervenciones breves y referencia al tratamiento por problemas de consumo de alcohol y otras drogas en hospitales que atienden personas que viven con el VIH/ SIDA en el Perú. Rev Peru Med Exp Salud Publica. 2016;33(3):432-7.

42. Gelberg L, Rey GN, Andersen RM, Arroyo M, Bojorquez-Chapela I, Rico MW, Vahidi M, Yacenda-Murphy $J$, Arangua L, Serota M. Prevalence of substance use among patients of community health centers in East Los Angeles and Tijuana. Subst Use Misuse. 2017;52(3):359-372.

43. Soto-Brandt G, et al. Evidencia de validez en Chile del Alcohol, Smoking and Substance Involvement Screening Test (ASSIST). Adicciones. 2013;26(4).

44. Costa PH, Mota DC, Cruvinel E, Paiva FS, Ronzani TM.. A methodology to implement preventive actions against harmful drug use in the context of PHC in Latin America. Rev Panam Salud Publica. 2013;33(5):325-331

45. Natera R et al. Final Narrative Report of the bi-national assist screening and quit using drugs intervention trial (quit) Tijuana/Los Angeles. Mexico: National Institute on Psychiatry de la Fuente Muniz;2014.

46. Kaner E. Brief alcohol interventions - time for translational research. Addiction 2010;105:960-961.

47. World Health Organization COVID-19 dashboard. https://covid19.who. int/. Accessed 5 Oct 2020

48. Shadish WR, Cook TD, Campbell DT. Experimental and quasiexperimental designs for generalized causal inference. 2nd edition. Michigan: Houghton Mifflin; 2002.

49. Anderson P, O'Donnell A, Kaner E et al. Scaling-up PHC-based prevention and management of alcohol use disorder at the municipal level in middle-income countries in Latin America: Background and preprotocol for a three-country quasi-experimental study. F1000Research. 2017;6:311. https://doi.org/10.12688/f1000research.11173.1.

50. Platt L, Melendez- Torres GJ, O'Donnell A, et al. How effective are brief interventions in reducing alcohol consumption: do the setting, practitioner group and content matter? Findings from a systematic review and metaregression analysis. BMJ Open. 2016;6:e011473. https://doi.org/ 10.1136/bmjopen-2016-011473.

51. Segura L, Anderson P, Gual A. Optimizing the delivery of interventions for harmful alcohol use in primary healthcare: an update. Curr Opin Psychiatry. 2018;31(4):324-332

52. Bush K, Kivlahan DR, McDonell MB, Fihn SD, Bradley KA. Ambulatory Care Quality Improvement P. The AUDIT alcohol consumption questions (AUDIT-C) - an effective brief screening test for problem drinking. Arch Intern Med. 1998;158(16):1789-95

53. PASS $^{16}$ sample size software. https://www.ncss.com/software/pass/: Donner, A. and Klar, N. 2000. Design and Analysis of Cluster Randomization Trials in Health Research. Arnold. London. Accessed 5 Oct 2020

54. R Core Team. R: A language and environment for statistical computing. $\mathrm{R}$ Foundation for Statistical Computing, Vienna; 2020. URL https://www. R-project.org/.

55. Mills KT, Bundy JD, Kelly TN, Reed JE, Kearney PM, Reynolds K, et al. Global disparities of hypertension prevalence and control: a systematic analysis of population-based studies from 90 countries. Circulation. 2016;134(6):441-50.

56. Chow CK, Teo KK, Rangarajan S, et al. PURE (Prospective Urban Rural Epidemiology) Study investigators. Prevalence, awareness, treatment, and control of hypertension in rural and urban communities in high-, middle-, and low-income countries. JAMA. 2013:310:959-968.

57. Lozano R, Soliz P, Gakidou E, et al. Benchmarking of performance of Mexican states with effective coverage. Lancet. 2006;368(9548):1729 1741. https://doi.org/10.1016/S0140-6736(06)69566-4.

58. Wensing $\mathbf{M}$, Huntink $\mathbf{E}$, van Lieshout $\mathbf{J}$, Godycki-Cwirko $\mathbf{M}$, Kowalczyk A, et al. Tailored Implementation of Evidence-Based Practice for Patients with Chronic Diseases. PLoS ONE. 2014;9(7):e101981. https://doi.org/10.1371/journal.pone.0101981.

59. Kaner EFS, Beyer FR, Garnett C, Crane D, Brown J, Muirhead C, Redmore J, O'Donnell A, Newham JJ, de Vocht F, Hickman M, Brown H, Maniatopoulos G, Michie S. Personalised digital interventions for reducing hazardous and harmful alcohol consumption in communitydwelling populations. Cochrane Database Syst Rev. 2017;(9): Art. No.: CD011479. https://doi.org/10.1002/14651858.CD011479.pub2.

Publisher's Note: Springer Nature remains neutral with regard to jurisdictional claims in published maps and institutional affiliations. 